\title{
Consumption of sugar-sweetened beverages and its association with nutrient intakes and diet quality in German children and adolescents
}

\author{
Lars Libuda $^{1 *}$, Ute Alexy ${ }^{1}$, Anette E. Buyken ${ }^{1}$, Wolfgang Sichert-Hellert ${ }^{1}$, Peter Stehle ${ }^{2}$ \\ and Mathilde Kersting ${ }^{1}$ \\ ${ }^{1}$ Research Institute of Child Nutrition (FKE), Heinstueck 11, D-44225 Dortmund, Germany \\ ${ }^{2}$ IEL - Nutritional Physiology, Rheinische Friedrich-Wilhelms-Universität Bonn, Bonn, Germany \\ (Received 18 April 2008 - Revised 3 September 2008 - Accepted 8 September 2008 - First published online 16 December 2008)
}

In the present study the relationship of sugar-sweetened beverage (SSB) consumption with the intake of single nutrients and total diet quality in German children and adolescents was evaluated using a repeated-measures regression analysis model. We used dietary data from 7145 three-day weighed records of 1069 subjects aged 2-19 years participating in the Dortmund Nutritional and Anthropometric Longitudinally Designed (DONALD) Study. Intake of macronutrients as percentage of total energy intake (\%En), intake of micronutrients as percentage of German reference values (intake quality score) and nutritional quality index (NQI) as an indicator of diet quality were chosen as separate dependent variables. SSB consumption was positively associated with \%En from carbohydrates (boys $v$. girls: $+4.00 v$. +4.09 En\%/MJ from SSB) and added sugars (boys $v$. girls: $+7.36 v .+9.52 \mathrm{En} \% / \mathrm{MJ}$ from SSB) and negatively with $\%$ En from protein (boys $v$. girls: $-1.25 v .-1.31 \mathrm{En} \% / \mathrm{MJ}$ from SSB) and fat (boys: $-2.82 v .-2.73$ En\%/MJ from SSB). With respect to micronutrients, SSB consumption was negatively associated with folate and $\mathrm{Ca}$ intake, for which mean intake levels were inadequate in girls. Absolute diet quality was negatively associated with SSB consumption, whereas the effect was larger for girls (boys $v$. girls: $-1.41 v .-2.63$ points of NQI/MJ from SSB). Overall, results show a diluting effect of SSB consumption on micronutrient intake and diet quality. This effect might be relevant especially in girls as the association with diet quality was larger and mean NQI levels were lower in comparison with boys.

Sugar-sweetened beverages: Diet quality: Nutrient intake: Children: Adolescents

Most previous studies on the consumption of sugar-sweetened beverages (SSB) have focused on its effect on total energy intake (TEI) and body weight. In fact, Malik et al. concluded in their recent review that greater consumption of sugar-sweetened drinks is associated with weight gain and obesity in both children and adults ${ }^{(1)}$. SSB are usually energy-dense, but have a relatively low micronutrient content ${ }^{(2)}$. Therefore, SSB consumption might have a diluting effect on micronutrient intake and total diet quality if SSB substitute nutrient-dense foods such as milk. Decreasing diet quality scores were found to increase disease risk and mortality rates in adulthood ${ }^{(3)}$. As eating and drinking habits are known to track between childhood and adulthood $^{(4)}$, the potential nutritional consequences of SSB consumption should already be considered in childhood and adolescence.

To date, the effect of SSB consumption on diet quality has predominantly been evaluated indirectly. SSB consumption has been associated with decreasing intakes of milk in children and adults ${ }^{(5)}$. Furthermore, Duffey \& Popkin observed a positive correlation between an unhealthy beverage pattern, characterised by a high consumption of sweetened drinks, and an unhealthy food pattern in adults ${ }^{(6)}$. In German children and adolescents, SSB were found to be one of the main contributors to added sugar intake ${ }^{(7)}$. An increasing intake of added sugars has been linked with decreasing intakes of micronutrients in several studies ${ }^{(7-10)}$. Additionally, a recent meta-analysis that was predominantly based on US-American studies observed a diluting effect of SSB consumption on $\mathrm{Ca}$ and riboflavin intake $^{(5)}$. Due to differences in beverage and food patterns between children in the USA and European countries, results from one country cannot necessarily be transferred to another. Furthermore, most previous studies focused on the association between SSB consumption and the intake of single nutrients, but did not evaluate the effect on total diet quality.

Therefore, the primary objective of this examination was to analyse the relationship of SSB consumption with intake of single nutrients and also total diet quality in a group of German adolescents from the Dortmund Nutritional and Anthropometric Longitudinally Designed (DONALD) Study. The total diet quality was estimated using the nutrient-based nutritional quality score (NQI). The secondary objective was to investigate possible sex and age interactions between SSB consumption and diet quality in order to identify subgroups at particular risk of dilution of total diet quality.

Abbreviations: DONALD, Dortmund Nutritional and Anthropometric Longitudinally Designed; IQS, intake quality score; MAR, mean adequacy ratio; NQI, nutritional quality index; SSB, sugar-sweetened beverage; TEI, total energy intake.

* Corresponding author: Lars Libuda, fax +49 2317115 81, email libuda@fke-do.de 


\section{Study design and methods}

Study design

The DONALD Study is an ongoing longitudinal, open cohort study that was established in 1985 at the Research Institute of Child Nutrition (Dortmund, Germany). The main aim of the study is the collection of information on nutrition, development, metabolism and health status of subjects between infancy and early adulthood. The regular assessments include $3 \mathrm{~d}$ weighed dietary records, anthropometry, urine sampling, as well as interviews on lifestyle and medical assessments. The regular visits begin at 3 months of age and take place annually from the age of 2 years until the age of 18 years. To date, over 1200 subjects have been enrolled in the DONALD Study; about forty subjects are joining each year. Further details of the DONALD Study are provided elsewhere ${ }^{(11)}$.

The DONALD Study is approved by the ethical committee of the Rheinische Friedrich-Wilhelms-Universität Bonn. All examinations and assessments are performed with parental and, later on, with the children's written consent.

\section{Subjects}

Dietary records ( $n$ 7740) of subjects aged between 2 and 19 years have been collected in the DONALD Study since 1985. Of those, we considered dietary records with a ratio of reported TEI and predicted individual $\mathrm{BMR}^{(12)}$ above the age- and sex-specific cut-off values, i.e. 0.97 for boys and girls aged 1-5 years; boys 1.04 and girls 1.01 aged 6-13 years; boys 1.07 and girls 0.97 aged $14-18$ years ${ }^{(13)}$. As a consequence, 506 records had to be excluded due to implausible dietary recording. Also, dietary records covering less than $3 \mathrm{~d}$ were excluded from evaluation. This selection resulted in a total of 7145 three-day dietary records from 1069 subjects (524 boys). The individual number of $3 \mathrm{~d}$ records ranged from one record ( $n$ 163) to seventeen records ( $n$ 18); mean number was 4.8 records per subject. Mean age was 8.0 (SD 4.5) years in boys and 8.0 (SD 4.4) years in girls.

\section{Dietary survey}

All foods and beverages before consumption as well as leftovers were weighed to the nearest $\mathrm{g}$ and recorded by the children's parents, or by the older subjects themselves, on three consecutive days using electronic food scales ( $3 \mathrm{~d}$ weighed dietary records). If weighing was not possible, semi-quantitative recording (for example, numbers of glasses, cups) was allowed. Weekdays $(69.8 \%)$ and weekend days $(30.2 \%)$ were proportionally distributed in the evaluated study sample.

Energy and nutrient intakes were calculated using our continuously updated in-house nutrient database LEBTAB, which contains detailed data on the energy and nutrient contents of all recorded food items ${ }^{(14)}$. The nutrient content of basic food items was taken from standard nutrient tables; the content of commercial food items was derived either from their product labels or from simulating recipes from the ingredients listed on the labels including fortified nutrients.

For distinction from low-energy beverages, SSB were defined as carbonated and uncarbonated beverages providing more than $40 \mathrm{~kJ} / 100 \mathrm{ml}$, the energy arising predominantly from added sugars (mono- and disaccharides). Since no universal definition existed to distinguish between lowenergy and regular SSB, we chose this cut-off based on information from our food database LEBTAB. Unfortunately, LEBTAB does not have information on added sugar for all SSB. However, carbohydrates in total provided more than $90 \%$ of total energy in more than $90 \%$ of all regular SSB. Therefore, SSB include lemonades, iced tea and fruit drinks (diluted and sugar-sweetened fruit juices). Juices made from $100 \%$ fruit and alcoholic beverages were not classified as SSB.

\section{Nutrient intake and diet quality}

For each $3 \mathrm{~d}$ record individual mean daily intakes of energy from added sugars and macronutrients (carbohydrates, fat, protein) and mean daily intake of vitamins and minerals (including micronutrients from fortified SSB) in three recorded days considering vitamin losses due to food preparation were calculated. Mean values of energy from macronutrients and added sugars were related to TEI. Added sugars were defined as all refined sugars (for example, sucrose, glucose, fructose, maltose), but also honey, syrup and sugar substitutes such as mannitol, eaten separately or as an ingredient in processed or prepared foods. For the particular vitamins and minerals, the intake quality score (IQS) $)^{(15,16)}$ was calculated as percentage of the latest age- and sex-specific dietary reference values for nutrient intakes of the German Nutrition Society ${ }^{(17)}$ :

$$
\mathrm{IQS}_{\mathrm{i}}=\frac{\text { mean intake }_{\mathrm{i}}}{\text { reference value }_{\mathrm{i}}} \times 100,
$$

where i denotes the particular nutrient.

We found two different definitions for nutrient adequacy in the literature, i.e. $77 \%{ }^{(10)}$ and $>$ two-thirds ${ }^{(9)}$ of the respective reference value. We decided to choose the more conservative cut-off value to determine nutrient inadequacy. Therefore, inadequate mean intake was defined as IQS $<$ two-thirds of the particular reference value ${ }^{(9)}$. IQS values of the following seventeen vitamins and minerals were considered separately:

(1) Vitamin A, vitamin $\mathrm{E}$, vitamin $\mathrm{K}$, thiamin, riboflavin, vitamin $\mathrm{B}_{6}$, vitamin $\mathrm{B}_{12}$, vitamin $\mathrm{C}$, niacin, pantothenic acid and folate;

(2) $\mathrm{Ca}, \mathrm{Mg}, \mathrm{Fe}, \mathrm{P}, \mathrm{K}$ and $\mathrm{Zn}$.

Since vitamin D values were not reliable for all foods in LEBTAB, we decided not to include vitamin $\mathrm{D}$ in our analysis. Some other nutrients (for example, Mn) were not included, because recommendations for these nutrients are only estimates.

The nutrient-based NQI was used to estimate the individual total diet quality ${ }^{(15,16)}$. NQI was calculated as the individual harmonic mean of each subject's IQS values of the seventeen micronutrients:

$$
\mathrm{NQI}=\frac{\mathrm{n}}{\sum_{i=1}^{n} \frac{1}{\mathrm{IQS}_{i}}} .
$$

To avoid mathematical compensation of deficient intakes of one nutrient by exceeding intakes of another nutrient, IQS values were truncated at 100 if the intake of a nutrient exceeded the reference value. NQI theoretically ranges from 
0 to 100 , where 100 represents a high diet quality meeting or exceeding the reference values in all considered micronutrients within three recorded days.

\section{Statistical analysis}

SAS procedures (version 8.02, 2001; SAS Institute, Inc., Cary, NC, USA) were applied for data analysis. Descriptive data are given as mean values and standard deviations, unless indicated otherwise.

The association between SSB consumption and diet quality was tested using a repeated-measures regression model (PROC MIXED; SAS Institute, Inc.). To concurrently regard absolute intake of SSB and differences in energy density of $\mathrm{SSB}$, energy (MJ) deriving from SSB was chosen as the parameter for SSB consumption and included as an independent variable. The percentage of energy from macronutrients, IQS values of single vitamins and minerals, and NQI were separately used as dependent variables. Model 1 was controlled for time since onset of the study and age of subjects. As TEI was not adjusted in model 1, this model analyses the association between SSB consumption and total nutrient intake. The coefficient $\beta$ in model 1 represents the predicted change in total nutrient intake (\% reference values or \% of TEI respectively) for each additional $1 \mathrm{MJ}$ of SSB consumption. In model 2, energy from SSB was adjusted for TEI using the residual method separately in particular age groups. Additionally, TEI was included as a covariable in model 2 . In this case, the association between SSB consumption and nutrient intake was estimated independently from TEI, i.e. model 2 analyses if a change in SSB consumption at a constant TEI level affects micronutrient intake. Since total nutrient intake depends on micronutrient density of a diet and TEI, the adjustment for TEI in model 2 is leading to prediction of the association between SSB consumption and micronutrient density.

Due to a significant sex interaction between SSB consumption and NQI in model 1 and model 2, we analysed boys and girls separately. We further tested for possible age interactions using two age groups (2-10 years and 11-19 years) to identify subgroups at particular risk of dilution of total diet quality. In all models, an exponential variance structure was assumed, which supposes a stronger correlation of assessments of one subject in two consecutive years than for longer time intervals. A separate model of repeated-measures regression analyses with time and age as sole independent variables was used for the description of time-adjusted age trends in energy and nutrient intake respectively for boys and girls (Tables 1 and 2). Subsequently, sex was included as an additional independent variable (Table 2). Positive coefficient values for sex suggest a higher nutrient intake in boys. In all statistical tests a $P$ value $<0.05$ was considered as significant.

\section{Results}

Sugar-sweetened beverage consumption and nutrient intakes

Mean values of daily SSB consumption and nutrient intake in boys and girls are shown in Tables 1 and 2. Mean intakes of most micronutrients met or even exceeded the reference values in both sexes. Inadequate mean intakes (IQS $<$ twothirds) were observed for folate and $\mathrm{Fe}$ in several age strata in girls and intake of $\mathrm{Ca}$ was borderline in older girls.
In boys, intake of folate was predominantly deficient in younger age groups.

\section{Sex differences and age trends in sugar-sweetened beverage consumption and nutrient intake}

TEI (MJ) and energy deriving from SSB (MJ) were higher in boys in comparison with girls (Table 2). Additionally, boys had higher IQS values of most vitamins and all minerals resulting in a higher NQI.

TEI and energy deriving from SSB increased with age in both sexes. However, TEI and energy from SSB increased faster with age in boys, resulting in the mentioned sex differences. The composition of macronutrients did not change substantially as boys grew older, whereas girls substituted to a moderate degree fat by carbohydrates. At the same time, the intake of added sugars increased with age in both sexes. While NQI increased with age in boys because of increasing IQS values in critical nutrients such as folate and Fe, NQI slightly decreased in girls mainly due to decreasing mean IQS values of various minerals.

\section{Association between sugar-sweetened beverage consumption and nutrient intake}

Results of the repeated-measures regression analysis of the association between SSB consumption and macronutrient intake are shown in Table 3. SSB consumption was positively associated with energy from carbohydrates in both sexes in model 1. However, the increase in energy from added sugars was higher than for total carbohydrates, i.e. energy from non-sugar carbohydrates decreased with increasing SSB consumption. Concomitant to the increase in energy from total carbohydrates the intake of protein and especially fat decreased as SSB consumption increased. Results were similar when SSB consumption was adjusted for TEI (model 2).

Results for micronutrients are shown in Table 4 . In contrast to macronutrients, the associations between SSB consumption and total micronutrient intakes were different between boys and girls (model 1). In boys, the intake of riboflavin, pantothenic acid, $\mathrm{Ca}, \mathrm{Fe}, \mathrm{P}$ and $\mathrm{K}$ significantly decreased by $6,5,6,6,5$ and $3 \%$ respectively in relation to reference values for each additional $1 \mathrm{MJ}$ of SSB consumption. The total intake of vitamin $\mathrm{E}$, niacin and vitamin $\mathrm{C}$, which are commonly fortified in SSB (Table 5), was positively associated with SSB consumption. In boys, NQI decreased only by 1.4 points for each $1 \mathrm{MJ}$ of SSB consumption. In girls, the intake of vitamin A, vitamin $\mathrm{K}$, riboflavin, folate, $\mathrm{Ca}, \mathrm{Mg}, \mathrm{P}$ and $\mathrm{K}$ significantly decreased by $9,37,8,5,9,10,6$, and $9 \%$ respectively for each additional $1 \mathrm{MJ}$ of SSB consumption in model 1. These lower nutrient intakes resulted in a decrease of 2.6 points in NQI for each MJ of SSB consumed. In model 1, the decrease in NQI for each $1 \mathrm{MJ}$ of SSB consumption was higher in girls. In model 2 , SSB consumption was negatively associated with the nutrient density of most vitamins and all minerals in both sexes. Also, energy-adjusted NQI decreased in boys and girls. However, in line with model 1, this decrease was larger in girls.

The association between SSB consumption and NQI in model 1 significantly differed between younger and older boys ( $P$ for age interaction $=0.006)$. In boys aged $2-10$ years, NQI decreased by 2.28 points for each $1 \mathrm{MJ}$ of SSB 
Table 1. Mean daily nutrient intakes and diet quality in 2- to 19-year-old male subjects of the Dortmund Nutritional and Anthropometric Longitudinally Designed Study (Mean values and standard deviations)

\begin{tabular}{|c|c|c|c|c|c|c|c|c|c|c|c|c|c|}
\hline \multirow[t]{2}{*}{ Age... } & \multicolumn{2}{|c|}{$2-4$ years } & \multicolumn{2}{|c|}{$5-7$ years } & \multicolumn{2}{|c|}{$8-10$ years } & \multicolumn{2}{|c|}{$11-13$ years } & \multicolumn{2}{|c|}{$14-15$ years } & \multicolumn{2}{|c|}{$16-19$ years } & \multirow[b]{2}{*}{$\beta \dagger$} \\
\hline & Mean & SD & Mean & SD & Mean & SD & Mean & SD & Mean & SD & Mean & SD & \\
\hline \multicolumn{14}{|c|}{ Sample characteristics } \\
\hline Subjects $(n)$ & \multicolumn{2}{|c|}{414} & \multicolumn{2}{|c|}{350} & \multicolumn{2}{|c|}{280} & \multicolumn{2}{|c|}{220} & \multicolumn{2}{|c|}{156} & \multicolumn{2}{|c|}{115} & \\
\hline Records $(n)$ & \multicolumn{2}{|c|}{982} & \multicolumn{2}{|c|}{850} & \multicolumn{2}{|c|}{693} & \multicolumn{2}{|c|}{507} & \multicolumn{2}{|c|}{252} & \multicolumn{2}{|c|}{265} & \\
\hline Age (years) & 3.0 & 0.8 & $6 \cdot 0$ & 0.8 & 9.0 & 0.8 & $12 \cdot 0$ & 0.8 & 14.5 & 0.5 & $17 \cdot 0$ & 0.8 & \\
\hline TEI $(M J / d)$ & 4.79 & 0.93 & $6 \cdot 30$ & 1.07 & 7.53 & 1.29 & $8 \cdot 67$ & 1.60 & $10 \cdot 48$ & $2 \cdot 12$ & $11 \cdot 27$ & 2.07 & $0.46^{\star \star \star}$ \\
\hline $\operatorname{SSB}(\mathrm{MJ} / \mathrm{d})$ & 0.11 & 0.21 & 0.21 & 0.30 & 0.31 & 0.37 & 0.47 & 0.52 & 0.67 & 0.73 & 0.74 & 0.75 & $0.05^{\star \star *}$ \\
\hline $\operatorname{SSB}(g / d)$ & 60.9 & 118.4 & $122 \cdot 2$ & $172 \cdot 6$ & $190 \cdot 6$ & 221.9 & 286.4 & 311.4 & $409 \cdot 1$ & 460.5 & $452 \cdot 8$ & 473.9 & $29 \cdot 3^{\star \star \star}$ \\
\hline \multicolumn{14}{|c|}{ Macronutrients (\% total energy intake) } \\
\hline Carbohydrates & $50 \cdot 7$ & 6.5 & $51 \cdot 1$ & 5.5 & $51 \cdot 1$ & 5.8 & $51 \cdot 3$ & $6 \cdot 0$ & $51 \cdot 8$ & $6 \cdot 0$ & $49 \cdot 8$ & $6 \cdot 4$ & -0.03 \\
\hline Added sugar & 11.5 & 5.6 & $14 \cdot 1$ & 5.5 & 14.4 & 5.6 & $15 \cdot 0$ & $6 \cdot 2$ & $15 \cdot 6$ & 6.8 & $13 \cdot 7$ & $6 \cdot 8$ & $0.31^{\star \star \star}$ \\
\hline Fat & $36 \cdot 3$ & $5 \cdot 8$ & $36 \cdot 3$ & $5 \cdot 0$ & $36 \cdot 0$ & $5 \cdot 3$ & $35 \cdot 6$ & $5 \cdot 7$ & $35 \cdot 1$ & $5 \cdot 6$ & $35 \cdot 2$ & $6 \cdot 2$ & -0.04 \\
\hline Protein & $12 \cdot 9$ & $2 \cdot 1$ & 12.5 & 1.8 & $12 \cdot 8$ & 1.9 & $13 \cdot 0$ & $2 \cdot 0$ & $13 \cdot 0$ & $2 \cdot 0$ & $13 \cdot 6$ & $2 \cdot 2$ & $0.02^{*}$ \\
\hline \multicolumn{14}{|c|}{ Micronutrients (\% reference value) } \\
\hline Vitamin A & $110 \cdot 3$ & $68 \cdot 8$ & $113 \cdot 3$ & $115 \cdot 2$ & $112 \cdot 4$ & $93 \cdot 6$ & 99.4 & 87.4 & $100 \cdot 7$ & $92 \cdot 7$ & 104.6 & 57.5 & -0.50 \\
\hline Vitamin E & 95.0 & $51 \cdot 6$ & $104 \cdot 2$ & $60 \cdot 6$ & $101 \cdot 1$ & $47 \cdot 7$ & $95 \cdot 1$ & $46 \cdot 3$ & $100 \cdot 9$ & 48.9 & $132 \cdot 4$ & 199.2 & $1.46^{\star * *}$ \\
\hline Vitamin K & $390 \cdot 0$ & $312 \cdot 8$ & 348.6 & 271.4 & $292 \cdot 7$ & 232.9 & 253.6 & 178.5 & 229.4 & 168.5 & $220 \cdot 4$ & 192.5 & $-12 \cdot 85^{\star \star \star}$ \\
\hline Thiamin & 104.7 & 53.3 & 104.7 & $47 \cdot 8$ & 103.3 & $43 \cdot 0$ & $100 \cdot 6$ & $51 \cdot 0$ & 101.3 & $39 \cdot 6$ & $117 \cdot 3$ & 50.1 & $0.52^{*}$ \\
\hline Riboflavin & $145 \cdot 1$ & $66 \cdot 4$ & $141 \cdot 2$ & $59 \cdot 2$ & $138 \cdot 2$ & 55.4 & $122 \cdot 2$ & $61 \cdot 1$ & $126 \cdot 6$ & $51 \cdot 8$ & 141.0 & 55.9 & $0.81^{\star \star}$ \\
\hline Niacin & $190 \cdot 3$ & $64 \cdot 0$ & 187.5 & $63 \cdot 8$ & 194.3 & $66 \cdot 2$ & $183 \cdot 8$ & $70 \cdot 7$ & 188.7 & $60 \cdot 9$ & $226 \cdot 3$ & $76 \cdot 1$ & $1 \cdot 30^{\star \star \star}$ \\
\hline Pantothenic acid & 69.0 & $32 \cdot 3$ & 83.9 & $36 \cdot 7$ & $86 \cdot 7$ & $40 \cdot 4$ & $97 \cdot 0$ & $98 \cdot 6$ & $91 \cdot 0$ & $37 \cdot 0$ & $109 \cdot 8$ & $127 \cdot 5$ & $2 \cdot 74^{\star \star \star}$ \\
\hline Vitamin $\mathrm{B}_{6}$ & 257.8 & $97 \cdot 0$ & $260 \cdot 3$ & $108 \cdot 4$ & $225 \cdot 7$ & $91 \cdot 6$ & $182 \cdot 6$ & $81 \cdot 0$ & $150 \cdot 6$ & $59 \cdot 3$ & $156 \cdot 6$ & 70.5 & $-8.08^{\star \star \star}$ \\
\hline Vitamin $B_{12}$ & 243.4 & 112.5 & $211 \cdot 7$ & $102 \cdot 2$ & 208.7 & $96 \cdot 6$ & 192.7 & $81 \cdot 1$ & $162 \cdot 7$ & $75 \cdot 3$ & $177 \cdot 7$ & 68.4 & $-4 \cdot 40^{\star \star \star}$ \\
\hline Folate & 64.5 & 33.5 & $65 \cdot 7$ & 39.1 & 73.7 & 32.9 & 67.6 & $32 \cdot 4$ & $77 \cdot 2$ & 31.5 & 85.5 & 41.9 & $1 \cdot 23^{* \star *}$ \\
\hline Vitamin C & 135.9 & 89.4 & $135 \cdot 3$ & $87 \cdot 6$ & $131 \cdot 7$ & $73 \cdot 0$ & $135 \cdot 7$ & $80 \cdot 4$ & $151 \cdot 1$ & 191.9 & $153 \cdot 7$ & $100 \cdot 7$ & 0.85 \\
\hline $\mathrm{Ca}$ & $101 \cdot 2$ & 34.7 & $98 \cdot 8$ & $32 \cdot 4$ & $92 \cdot 8$ & 30.9 & 87.5 & $30 \cdot 0$ & $97 \cdot 7$ & 38.9 & $109 \cdot 0$ & 38.6 & $-0.38^{*}$ \\
\hline $\mathrm{Mg}$ & 201.0 & $59 \cdot 6$ & 168.9 & 48.0 & 144.8 & 41.5 & 119.6 & $31 \cdot 8$ & 105.4 & $32 \cdot 8$ & 99.6 & 29.5 & $-7 \cdot 80^{\star \star \star}$ \\
\hline $\mathrm{Fe}$ & $69 \cdot 1$ & $23 \cdot 0$ & $88 \cdot 2$ & $82 \cdot 3$ & $82 \cdot 2$ & $22 \cdot 3$ & 81.9 & $23 \cdot 0$ & $97 \cdot 0$ & $36 \cdot 7$ & $110 \cdot 7$ & $105 \cdot 3$ & $2 \cdot 21^{\star \star \star}$ \\
\hline$P$ & $145 \cdot 2$ & $36 \cdot 3$ & $146 \cdot 3$ & $37 \cdot 3$ & 130.1 & $36 \cdot 3$ & $101 \cdot 6$ & $24 \cdot 7$ & 121.5 & 33.1 & 134.6 & 33.9 & $-2 \cdot 07^{\star \star \star}$ \\
\hline $\mathrm{K}$ & 164.7 & 41.8 & $143 \cdot 4$ & 33.4 & $150 \cdot 6$ & 35.8 & $157 \cdot 0$ & 38.5 & 167.6 & 47.4 & $173 \cdot 3$ & $46 \cdot 4$ & 0.20 \\
\hline $\mathrm{Zn}$ & $158 \cdot 3$ & $90 \cdot 9$ & $125 \cdot 5$ & $105 \cdot 3$ & $112 \cdot 7$ & $104 \cdot 6$ & $102 \cdot 7$ & $58 \cdot 3$ & 114.0 & 31.9 & $125 \cdot 9$ & $49 \cdot 1$ & $-3 \cdot 49^{\star \star \star}$ \\
\hline \multicolumn{14}{|l|}{ Diet quality } \\
\hline NQI & $83 \cdot 2$ & $10 \cdot 0$ & $85 \cdot 6$ & $9 \cdot 6$ & $86 \cdot 2$ & 9.9 & 83.9 & 11.1 & $85 \cdot 8$ & 11.6 & $89 \cdot 1$ & $9 \cdot 7$ & $0.24^{\star \star \star}$ \\
\hline
\end{tabular}

TEl, total energy intake; SSB, sugar-sweetened beverages; NQI, Nutritional Quality Index.

$\dagger$ Results from repeated-measures regression analyses on the time-adjusted effect of age (years) on the intake of single nutrients. 
Table 2. Mean daily nutrient intakes and diet quality in 2- to 19-year-old female subjects of the Dortmund Nutritional and Anthropometric Longitudinally Designed Study (Mean values and standard deviations)

\begin{tabular}{|c|c|c|c|c|c|c|c|c|c|c|c|c|c|c|}
\hline \multirow[t]{2}{*}{ Age... } & \multicolumn{2}{|c|}{$2-4$ years } & \multicolumn{2}{|c|}{$5-7$ years } & \multicolumn{2}{|c|}{$8-10$ years } & \multicolumn{2}{|c|}{$11-13$ years } & \multicolumn{2}{|c|}{$14-15$ years } & \multicolumn{2}{|c|}{$16-19$ years } & \multirow[b]{2}{*}{$\beta$ Age† } & \multirow[b]{2}{*}{$\beta$ Sex $\ddagger$} \\
\hline & Mean & SD & Mean & SD & Mean & SD & Mean & SD & Mean & SD & Mean & SD & & \\
\hline \multicolumn{15}{|c|}{ Sample characteristics } \\
\hline Subjects $(n)$ & \multirow{2}{*}{\multicolumn{2}{|c|}{$\begin{array}{c}437 \\
1017\end{array}$}} & \multirow{2}{*}{\multicolumn{2}{|c|}{$\begin{array}{l}353 \\
861\end{array}$}} & \multicolumn{2}{|c|}{295} & \multirow{2}{*}{\multicolumn{2}{|c|}{$\begin{array}{l}231 \\
536\end{array}$}} & \multirow{2}{*}{\multicolumn{2}{|c|}{$\begin{array}{l}140 \\
221\end{array}$}} & \multirow{2}{*}{\multicolumn{2}{|c|}{$\begin{array}{l}106 \\
242\end{array}$}} & & \\
\hline Records $(n)$ & & & & & & & & & & & & & & \\
\hline Age (years) & 3.0 & 0.8 & $6 \cdot 0$ & 0.8 & $9 \cdot 0$ & 0.8 & $12 \cdot 0$ & 0.8 & 14.5 & 0.5 & $17 \cdot 0$ & 0.8 & & \\
\hline TEI ( $\mathrm{MJ} / \mathrm{d})$ & 4.38 & 0.80 & 5.66 & 0.97 & $6 \cdot 65$ & $1 \cdot 16$ & 7.55 & 1.32 & 8.09 & 1.46 & $8 \cdot 16$ & 1.38 & $0.31^{* \star *}$ & $0.92^{\star \star *}$ \\
\hline$S S B(M J / d)$ & 0.09 & 0.19 & 0.17 & 0.25 & 0.24 & 0.32 & 0.36 & 0.46 & 0.44 & 0.48 & 0.42 & 0.58 & $0.03^{\star \star \star}$ & $0.08^{\star \star \star}$ \\
\hline SSB $(g / d)$ & 53.3 & $114 \cdot 2$ & $95 \cdot 4$ & 143.8 & 143.3 & $194 \cdot 3$ & $218 \cdot 2$ & 272.6 & 260.8 & 283.4 & $247 \cdot 0$ & 319.4 & $15 \cdot 9^{* \star \star}$ & $51 \cdot 4^{\star \star \star}$ \\
\hline \multicolumn{15}{|c|}{ Macronutrients (\% total energy intake) } \\
\hline Carbohydrates & 50.0 & 6.5 & $51 \cdot 3$ & 5.8 & $51 \cdot 3$ & $5 \cdot 8$ & $51 \cdot 2$ & $6 \cdot 1$ & 51.5 & 6.5 & $52 \cdot 3$ & $6 \cdot 2$ & $0.06^{\star}$ & 0.01 \\
\hline Added sugar & 11.4 & $5 \cdot 6$ & $14 \cdot 2$ & $5 \cdot 7$ & $14 \cdot 6$ & 5.9 & $14 \cdot 6$ & 6.5 & 14.9 & 6.9 & 13.9 & $6 \cdot 4$ & $0.25^{\star \star \star}$ & 0.20 \\
\hline Fat & $36 \cdot 8$ & $5 \cdot 8$ & $36 \cdot 1$ & $5 \cdot 2$ & $36 \cdot 0$ & $5 \cdot 3$ & $35 \cdot 7$ & $5 \cdot 6$ & 35.4 & $5 \cdot 7$ & 34.2 & $5 \cdot 7$ & $-0.06^{\star}$ & -0.11 \\
\hline Protein & $13 \cdot 1$ & $2 \cdot 1$ & $12 \cdot 4$ & $2 \cdot 0$ & $12 \cdot 6$ & $2 \cdot 0$ & $13 \cdot 0$ & $2 \cdot 2$ & $12 \cdot 9$ & $2 \cdot 2$ & $13 \cdot 1$ & $2 \cdot 0$ & -0.01 & 0.04 \\
\hline \multicolumn{15}{|c|}{ Micronutrients (\% reference value) } \\
\hline Vitamin A & 102.9 & $70 \cdot 7$ & $102 \cdot 1$ & $74 \cdot 7$ & $100 \cdot 2$ & $72 \cdot 7$ & $105 \cdot 1$ & $108 \cdot 6$ & 97.5 & $77 \cdot 0$ & $113 \cdot 2$ & $102 \cdot 9$ & 0.67 & 4.81 \\
\hline Vitamin E & $105 \cdot 1$ & 57.9 & $95 \cdot 1$ & $45 \cdot 6$ & $100 \cdot 9$ & $52 \cdot 3$ & $101 \cdot 7$ & 52.7 & 98.0 & 48.7 & $106 \cdot 7$ & $52 \cdot 3$ & -0.29 & -0.58 \\
\hline Vitamin $\mathrm{K}$ & 365.5 & 299.5 & 329.4 & $267 \cdot 7$ & $275 \cdot 0$ & 237.4 & 239.6 & $168 \cdot 7$ & 249.5 & 300.5 & $197 \cdot 1$ & 189.9 & $-12 \cdot 87^{\star \star \star}$ & $16 \cdot 47$ \\
\hline Thiamin & $96 \cdot 5$ & $43 \cdot 1$ & $96 \cdot 2$ & $56 \cdot 7$ & $96 \cdot 4$ & $55 \cdot 3$ & $105 \cdot 1$ & $52 \cdot 8$ & $100 \cdot 8$ & $40 \cdot 3$ & $117 \cdot 6$ & $102 \cdot 3$ & $0.92^{\star *}$ & $4 \cdot 28^{*}$ \\
\hline Riboflavin & $136 \cdot 1$ & $56 \cdot 4$ & $126 \cdot 3$ & $63 \cdot 6$ & 123.3 & $65 \cdot 7$ & 123.3 & 59.6 & $117 \cdot 1$ & $45 \cdot 9$ & 133.7 & 83.8 & $-1.03^{\star \star}$ & $8 \cdot 83^{\star \star *}$ \\
\hline Niacin & 178.9 & 62.5 & $167 \cdot 4$ & $56 \cdot 7$ & $173 \cdot 2$ & $62 \cdot 1$ & 184.1 & 63.9 & $178 \cdot 3$ & 61.6 & $206 \cdot 3$ & $76 \cdot 8$ & $0.73^{*}$ & $12 \cdot 94^{\star \star *}$ \\
\hline Pantothenic acid & 69.9 & 72.5 & $75 \cdot 7$ & 34.0 & 74.0 & 34.5 & 97.6 & $327 \cdot 8$ & $71 \cdot 6$ & $27 \cdot 2$ & $83 \cdot 3$ & $101 \cdot 3$ & $1.33^{*}$ & $7 \cdot 70^{\star \star}$ \\
\hline Vitamin $\mathrm{B}_{6}$ & 237.9 & $95 \cdot 4$ & 235.7 & 97.4 & $200 \cdot 4$ & $95 \cdot 2$ & 161.3 & $76 \cdot 3$ & $130 \cdot 1$ & $60 \cdot 4$ & $157 \cdot 2$ & 93.6 & $-8 \cdot 12^{\star * *}$ & $18 \cdot 49^{\star * \star}$ \\
\hline Vitamin $B_{12}$ & 230.5 & $106 \cdot 6$ & $182 \cdot 5$ & $77 \cdot 8$ & 179.5 & $97 \cdot 1$ & 174.0 & $102 \cdot 3$ & $120 \cdot 0$ & $52 \cdot 1$ & $160 \cdot 6$ & $567 \cdot 1$ & $-5 \cdot 28^{\star \star \star}$ & $22.53^{\star \star \star}$ \\
\hline Folate & $62 \cdot 3$ & $31 \cdot 7$ & 58.5 & 28.4 & $68 \cdot 8$ & 41.9 & 61.3 & 32.9 & $62 \cdot 2$ & 29.5 & $66 \cdot 2$ & 31.9 & 0.20 & $5 \cdot 56^{\star \star}$ \\
\hline Vitamin C & $122 \cdot 0$ & $75 \cdot 9$ & $124 \cdot 2$ & $73 \cdot 1$ & $130 \cdot 3$ & $76 \cdot 6$ & 130.9 & 77.6 & 128.5 & 82.5 & $133 \cdot 0$ & $79 \cdot 2$ & 0.55 & $8 \cdot 26^{\star \star}$ \\
\hline $\mathrm{Ca}$ & $95 \cdot 0$ & $33 \cdot 3$ & $87 \cdot 0$ & $27 \cdot 9$ & $79 \cdot 2$ & 27.5 & $74 \cdot 2$ & $25 \cdot 7$ & 73.6 & $25 \cdot 3$ & 77.4 & 24.9 & $-1.89^{\star \star \star}$ & $12 \cdot 15^{\star \star \star}$ \\
\hline $\mathrm{Mg}$ & $183 \cdot 3$ & 49.5 & 149.5 & 38.6 & $125 \cdot 7$ & 34.1 & $100 \cdot 2$ & $25 \cdot 5$ & 85.4 & 23.7 & 84.0 & $22 \cdot 1$ & $-8.09^{\star \star \star}$ & $17 \cdot 74^{\star \star \star}$ \\
\hline $\mathrm{Fe}$ & $69 \cdot 6$ & $128 \cdot 3$ & $75 \cdot 2$ & $24 \cdot 1$ & $70 \cdot 2$ & 21.4 & 59.4 & $23 \cdot 6$ & $62 \cdot 3$ & $20 \cdot 2$ & 63.8 & 19.5 & $-0.84^{\star}$ & $13 \cdot 71^{\star \star \star}$ \\
\hline$P$ & 134.4 & 33.6 & 129.1 & 31.3 & 111.5 & 31.6 & $87 \cdot 8$ & 20.4 & 92.9 & 23.7 & $95 \cdot 2$ & $21 \cdot 1$ & $-3 \cdot 78^{\star \star \star}$ & $16 \cdot 86^{\star \star \star}$ \\
\hline $\mathrm{K}$ & 149.5 & 38.8 & 129.1 & $30 \cdot 3$ & 133.6 & 30.9 & 140.3 & 34.8 & $132 \cdot 6$ & 32.6 & 134.4 & 33.3 & $-1.06^{\star * *}$ & $16 \cdot 99^{\star \star \star}$ \\
\hline $\mathrm{Zn}$ & 141.9 & $46 \cdot 0$ & $107 \cdot 3$ & $32 \cdot 1$ & $101 \cdot 7$ & 70.0 & 116.4 & 36.6 & $121 \cdot 7$ & 34.0 & $127 \cdot 0$ & 53.9 & $-1.68^{\star \star \star}$ & $8 \cdot 61^{\star *}$ \\
\hline \multicolumn{15}{|l|}{ Diet quality } \\
\hline NQI & 81.6 & $10 \cdot 0$ & 82.4 & $10 \cdot 3$ & $81 \cdot 6$ & $10 \cdot 9$ & 79.5 & 11.7 & $78 \cdot 1$ & $12 \cdot 8$ & $81 \cdot 2$ & $10 \cdot 9$ & $-0.20^{* *}$ & $3.03^{\star \star *}$ \\
\hline
\end{tabular}

TEI, total energy intake; SSB, sugar-sweetened beverages; NQI, Nutritional Quality Index.

${ }^{*} P<0.05,{ }^{* *} P<0.01,{ }^{* \star *} P<0.0001$.

(the time-adjusted effect of age (years) on the intake of single nutrients.

¥Results from repeated-measures regression analyses on the age- and time-adjusted effect of sex on the intake of single nutrients; positive values indicate a higher nutrient intake in boys. 
Table 3. Results of the mixed linear regression model of the association between sugar-sweetened beverage (SSB) consumption and intake of macronutrients in a sample of the Dortmund Nutritional and Anthropometric Longitudinally Designed Study

\begin{tabular}{|c|c|c|c|c|c|c|c|c|c|c|}
\hline \multirow{4}{*}{$\begin{array}{l}\text { Macronutrients } \\
\text { (\% total energy intake) }\end{array}$} & \multicolumn{5}{|c|}{ Model $1^{*}$} & \multicolumn{5}{|c|}{ Model 2† } \\
\hline & \multicolumn{4}{|c|}{ MJ from SSB } & \multirow{3}{*}{$\begin{array}{c}\text { Sex interaction } \\
(P)\end{array}$} & \multicolumn{4}{|c|}{ MJ from SSB } & \multirow{3}{*}{$\begin{array}{l}\text { Sex interaction } \\
(P)\end{array}$} \\
\hline & \multicolumn{2}{|c|}{ Boys } & \multicolumn{2}{|c|}{ Girls } & & \multicolumn{2}{|c|}{ Boys } & \multicolumn{2}{|c|}{ Girls } & \\
\hline & $\beta$ & $P$ & $\beta$ & $P$ & & $\beta$ & $P$ & $\beta$ & $P$ & \\
\hline Carbohydrates & 4.00 & $<0.0001$ & 4.09 & $<0.0001$ & 0.036 & 4.41 & $<0.0001$ & 4.54 & $<0.0001$ & 0.658 \\
\hline Added sugar & $7 \cdot 36$ & $<0.0001$ & 9.52 & $<0.0001$ & $<0.0001$ & $7 \cdot 72$ & $<0.0001$ & $9 \cdot 61$ & $<0.0001$ & $<0.0001$ \\
\hline Fat & $-2 \cdot 82$ & $<0.0001$ & $-2 \cdot 73$ & $<0.0001$ & 0.541 & $-3 \cdot 25$ & $<0.0001$ & -3.28 & $<0.0001$ & 0.818 \\
\hline Protein & -1.25 & $<0.0001$ & -1.31 & $<0.0001$ & 0.005 & $-1 \cdot 20$ & $<0.0001$ & -1.20 & $<0.0001$ & 0.928 \\
\hline
\end{tabular}

${ }^{*}$ Adjusted for time and age.

† Adjusted for time, age and total energy intake.

consumed $(P \leq 0 \cdot 001)$, while the NQI did not significantly decrease in boys aged $11-19$ years $(\beta=-0 \cdot 77 ; P=0 \cdot 149)$. In girls, there was no significant difference in the association between SSB consumption and NQI in model 1 between younger and older girls ( $P$ for age interaction $=0 \cdot 86$ ).

\section{Discussion}

In the present examination a negative association between SSB consumption and the total intake of various nutrients was observed which resulted in a lower total diet quality (model 1). The diluting effect of SSB consumption on diet quality was larger in girls than in boys. In addition, SSB consumption at a constant level of TEI was adversely associated with the intake of many micronutrients and diet quality in both sexes in the energy-adjusted model 2 . Thus, the supposed diluting effect of SSB on diet quality was confirmed in a sample of German children and adolescents.

In girls SSB consumption was negatively associated with the total intake of various nutrients in model 1. Therefore, SSB apparently displaced more nutritious foods or were associated with a high consumption of other nutrient-poor foods. Most remarkable was the negative effect on folate and $\mathrm{Ca}$ due to inadequate mean intakes of these nutrients in various age groups in girls and to their supposed effects in the prevention of $\mathrm{CVD}^{(18,19)}$ and on bone health ${ }^{(20)}$. Our

Table 4. Results of the mixed linear regression model of the association between sugar-sweetened beverage (SSB) consumption and intake of micronutrients in a sample of the Dortmund Nutritional and Anthropometric Longitudinally Designed Study

\begin{tabular}{|c|c|c|c|c|c|c|c|c|c|c|}
\hline \multirow{4}{*}{$\begin{array}{l}\text { IQS values } \\
\text { (\% reference value) }\end{array}$} & \multicolumn{5}{|c|}{ Model 1 (total nutrient intake) ${ }^{*}$} & \multicolumn{5}{|c|}{ Model 2 (nutrient density) $\dagger$} \\
\hline & \multicolumn{4}{|c|}{ MJ from SSB } & \multirow{3}{*}{$\begin{array}{c}\text { Sex interaction } \\
(P)\end{array}$} & \multicolumn{4}{|c|}{ MJ from SSB } & \multirow{3}{*}{$\begin{array}{c}\text { Sex interactio } \\
(P)\end{array}$} \\
\hline & \multicolumn{2}{|c|}{ Boys } & \multicolumn{2}{|c|}{ Girls } & & \multicolumn{2}{|c|}{ Boys } & \multicolumn{2}{|c|}{ Girls } & \\
\hline & $\beta$ & $P$ & $\beta$ & $P$ & & $\beta$ & $P$ & $\beta$ & $P$ & \\
\hline \multicolumn{11}{|l|}{ Vitamins } \\
\hline Vitamin A & -4.56 & 0.241 & -8.67 & 0.047 & 0.763 & -11.04 & 0.006 & -14.88 & $<0.001$ & 0.599 \\
\hline Vitamin E & $17 \cdot 11$ & $<0.0001$ & $5 \cdot 30$ & 0.063 & $<0.0001$ & $7 \cdot 81$ & 0.009 & $-4 \cdot 19$ & 0.138 & 0.003 \\
\hline Vitamin $\mathrm{K}$ & -9.57 & 0.377 & -36.90 & 0.008 & 0.489 & -24.41 & 0.029 & -52.07 & $<0.001$ & 0.245 \\
\hline Thiamin & -0.57 & 0.784 & 1.37 & 0.652 & 0.281 & -7.98 & $<0.0001$ & -8.28 & 0.006 & 0.942 \\
\hline Riboflavin & -6.03 & 0.019 & -7.70 & 0.022 & 0.594 & $-15 \cdot 39$ & $<0.0001$ & -19.95 & $<0.0001$ & 0.264 \\
\hline Niacin & 5.63 & 0.048 & 0.218 & 0.949 & 0.136 & -7.64 & 0.001 & $-15 \cdot 31$ & $<0.0001$ & 0.086 \\
\hline Pantothenic acid & $-5 \cdot 21$ & 0.045 & -3.00 & 0.671 & 0.341 & $-11 \cdot 70$ & $<0.0001$ & $-15 \cdot 11$ & 0.036 & 0.477 \\
\hline Vitamin $B_{6}$ & $-2 \cdot 34$ & 0.562 & -4.95 & 0.320 & 0.922 & $-12 \cdot 78$ & 0.002 & -18.80 & $<0.001$ & 0.435 \\
\hline Vitamin $B_{12}$ & -7.63 & 0.063 & $19 \cdot 62$ & 0.028 & 0.068 & $-21 \cdot 70$ & $<0.0001$ & $1 \cdot 10$ & 0.902 & 0.024 \\
\hline Folate & -0.83 & 0.587 & $-5 \cdot 27$ & 0.004 & 0.002 & -5.28 & $<0.001$ & $-10 \cdot 32$ & $<0.001$ & 0.086 \\
\hline Vitamin C & $9 \cdot 18$ & 0.027 & -0.06 & 0.988 & 0.250 & 0.31 & 0.941 & -9.60 & 0.016 & 0.184 \\
\hline \multicolumn{11}{|l|}{ Minerals } \\
\hline $\mathrm{Ca}$ & -6.35 & $<0.0001$ & -9.44 & $<0.0001$ & $<0.0001$ & -14.56 & $<0.0001$ & $-16 \cdot 66$ & $<0.0001$ & 0.480 \\
\hline $\mathrm{Mg}$ & -3.01 & 0.096 & -9.73 & $<0.0001$ & 0.020 & -13.50 & $<0.0001$ & $-19 \cdot 18$ & $<0.0001$ & 0.209 \\
\hline $\mathrm{Fe}$ & -5.80 & 0.012 & -4.98 & 0.196 & 0.002 & $-13 \cdot 15$ & $<0.0001$ & -10.79 & 0.006 & 0.589 \\
\hline$P$ & -4.74 & $<0.001$ & -5.98 & $<0.001$ & 0.003 & -15.66 & $<0.0001$ & -15.44 & $<0.0001$ & 0.720 \\
\hline $\mathrm{K}$ & -3.11 & 0.046 & -8.94 & $<0.0001$ & $<0.0001$ & -15.94 & $<0.0001$ & -20.60 & $<0.0001$ & 0.088 \\
\hline $\mathrm{Zn}$ & 0.79 & 0.840 & -4.91 & 0.071 & 0.973 & $-12 \cdot 40$ & 0.002 & $-15 \cdot 90$ & $<0.0001$ & 0.303 \\
\hline \multicolumn{11}{|l|}{ Diet quality } \\
\hline $\mathrm{NQI}$ & -1.41 & $<0.001$ & -2.63 & $<0.0001$ & $<0.0001$ & -4.08 & $<0.0001$ & $-6 \cdot 26$ & $<0.0001$ & $<0.001$ \\
\hline
\end{tabular}

IQS, Intake Quality Score; NQI, Nutritional Quality Index.

${ }^{*}$ Adjusted for time and age.

†Adjusted for time, age and total energy intake. 
Table 5. Fortification of micronutrients in sugar-sweetened beverages (SSB)

\begin{tabular}{lc}
\hline & \% of SSB \\
\hline Fortified micronutrients $(n)$ & \\
0 & $37 \cdot 9$ \\
1 & $17 \cdot 7$ \\
$2-5$ & $16 \cdot 1$ \\
$6-10$ & $19 \cdot 6$ \\
$>10$ & $8 \cdot 8$ \\
Top five of fortified micronutrients & \\
Vitamin C & $55 \cdot 6$ \\
Vitamin E & 32.4 \\
Vitamin $B_{6}$ & $31 \cdot 0$ \\
Vitamin A & 28.4 \\
Folate & $26 \cdot 1$ \\
\hline
\end{tabular}

findings in girls on folate and $\mathrm{Ca}$ intake are in line with results from previous studies. Especially in the case of $\mathrm{Ca}$, most studies in children and adolescents found a significantly lower intake in high consumers of $\mathrm{SSB}^{(2,21-27)}$, while one study found no association ${ }^{(28)}$ and another a positive association $^{(9)}$. In a recent meta-analysis, the authors conclude that there is a moderate negative effect of SSB consumption on $\mathrm{Ca}$ intake in children ${ }^{(5)}$. In terms of folate intake, two studies also found a negative association with SSB consumption ${ }^{(21,26)}$, whereas one study did not find any association ${ }^{(22)}$. Beside these two nutrients, we found negative associations with intakes of some other micronutrients such as vitamin $\mathrm{K}$, $\mathrm{Mg}$ and riboflavin. A meta-analysis supports this finding for riboflavin ${ }^{(5)}$. However, the mean intake of riboflavin as well as vitamin $\mathrm{K}$ exceeded the reference values in girls of the present study sample and should not get critical even in high consumers of SSB. As IQS values were truncated at 100 , the negative association with riboflavin and vitamin $\mathrm{K}$ intake had no effect on NQI values as an indicator for total diet quality. Regarding all micronutrients, the present examination suggests that absolute diet quality decreased with increasing SSB consumption in girls and to smaller degree in boys. So far, the few, mostly cross-sectional, studies on the effect of SSB consumption on total diet quality support the supposed negative effect of SSB consumption on total diet quality. Two studies found a negative association with total diet quality in younger children aged between 1 and 7 years using the food-based healthy eating index ${ }^{(23)}$ or the nutrient-based mean adequacy ratio (MAR) ${ }^{(27)}$. A significant lower diet quality measured by the healthy eating index was also observed in high soda consumers between 6 and 11 years of age ${ }^{(29)}$. In contrast, a weak positive effect of SSB consumption on the healthy eating index was found in a cross-sectional analysis in a population ranging from childhood to adulthood ${ }^{(30)}$. LaRowe et al. concluded that diet quality of all children could benefit from decreasing soda and sweetened beverage consumption ${ }^{(29)}$. Our findings suggest that this conclusion could be transferred to adolescence at least in girls.

In boys, SSB consumption was negatively associated with the intake of riboflavin, pantothenic acid, $\mathrm{Ca}, \mathrm{Fe}, \mathrm{P}$ and $\mathrm{K}$. These negative associations resulted in a decrease in absolute NQI. Due to positive associations with the intake of vitamin $\mathrm{E}$, niacin and vitamin $\mathrm{C}$, which are commonly fortified in $\mathrm{SSB}$, in this examination the decrease in NQI was smaller in boys in comparison with girls. Here, the diluting effect of SSB consumption on total diet quality was reduced by fortification. Earlier results from the DONALD Study demonstrated that fortification had a masking effect on the nutrient dilution of added sugars ${ }^{(31)}$. Since total diet quality was just slightly impaired in boys, SSB probably did not widely displace other foods in diets, but was drunken additionally. This resulted in nearly unaffected total nutrient intake (model 1), but decreasing nutrient density (model 2) due to higher TEI. Actually, a previous analysis of the DONALD Study found no adequate compensation for energy from SSB by restriction of other energetic foods resulting in higher $\mathrm{TEI}^{(32)}$. Although the effect of SSB consumption on absolute diet quality is small in boys, the diluting effect on nutrient density, the concomitant increase in TEI and the consequences for energy balance are unfavourable.

In girls the diluting effect of SSB consumption was larger in comparison with boys. In contrast to boys, the negative association was still significant as girls grew older. Since mean NQI decreased with age in girls, high SSB consumption in adolescent girls in particular might be cause for concern.

For evaluation of total diet quality, we used the nutrientbased NQI. Another common nutrient-based score is the $\operatorname{MAR}^{(33,34)}$, which is also based on single nutrient intake levels given as a percentage of reference values (i.e. IQS). As values of single nutrients were truncated at 100, NQI and MAR are unaffected by nutrients which exceeded the reference values. In contrast to NQI, which is the harmonic mean of the IQS values, MAR is calculated as the arithmetic mean. One advantage of the harmonic mean is that it is more sensitive to imbalances in nutrient intake than the arithmetic mean. As a consequence, a low intake of one nutrient is not as easily compensated for by a high intake of another nutrient ${ }^{(15)}$. Gedrich et al. concluded that the NQI is likely to be a more sensitive instrument to classify a deficient food quality $^{(16)}$. However, when using MAR instead of NQI as the dependent variable in our sample, the consumption of SSB similarly affected diet quality in girls (results not shown). As intake levels of only a few micronutrients were affected in boys, there was no significant relationship between SSB consumption and MAR in model 1. Therefore, NQI is more appropriate for evaluation of the effect of SSB consumption on diet quality due to higher sensitivity for deficiency nutrients.

In terms of macronutrients, the present results (Table 3) confirm findings of various studies that observed higher carbohydrate and lower protein intakes in relation to TEI in high consumers of $\mathrm{SSB}^{(2,5,9,21,22,35)}$. In the present examination, the relative increase in added sugars was higher than the increase of all carbohydrates, i.e. relative intake of nonsugar carbohydrates was lower in those who consumed more SSB. A meta-analysis also primarily attributed the observed increase in carbohydrate intake in SSB consumers to the greater consumption of added sugar ${ }^{(5)}$. Findings on the relationship with fat intake were less consistent ${ }^{(2,5,9,21,35)}$.

The DONALD Study has various strengths, but also limitations that need to be mentioned. Information on dietary habits and food intake are self-reported and might be influenced by recording errors and under-reporting. However, we excluded obviously implausible records. Most other studies on beverage 
consumption and nutrient intake used either single $24 \mathrm{~h}$ recalls or FFQ, both of which are prone to misjudge portion size and therefore total nutrient intake in children and adolescents in contrast to weighed dietary records. The high standard of the dietary records in the DONALD Study has recently been shown in an analysis comparing dietary iodine intake from weighed amounts of different food groups with concurrent $24 \mathrm{~h}$ urinary iodine excretion ${ }^{(36)}$. The elaborate design of the DONALD Study results in a relative small study sample, which is not representative with respect to socio-economic status. Nevertheless, a recent nationwide survey observed similar micronutrient intakes in German children and adolescents $^{(37)}$. Additionally, an earlier nationwide German dietary survey from 1987-8 observed no or only minor differences in dietary habits in comparison with the DONALD sample ${ }^{(38,39)}$. Therefore, our selected sample appears to reflect common patterns of nutrient intakes in German children and adolescents.

\section{Conclusion}

In a sample of German children and adolescents, the consumption of SSB was negatively associated with total diet quality in boys and girls. These findings suggest that SSB consumption might decrease mean diet quality despite an adequate mean intake of most micronutrients. However, diet quality of girls suffered more from SSB consumption. Most remarkable were the negative effects on the already low folate and $\mathrm{Ca}$ intake. As food and beverage patterns might track from adolescence to adulthood, the present results suggest that SSB consumption should preferably be limited in childhood in order to prevent lasting dilution effects on diet quality.

\section{Acknowledgements}

The present study was supported and funded by the German Federal Ministry of Food, Agriculture and Consumer Protection. The DONALD Study is further supported by the Ministry of Education, Science and Research North Rhine-Westphalia, Germany.

L. L. conducted the statistical analyses and wrote the manuscript. M. K., L. L. and U. A. conceived of the research project. W. S.-H. provided technical support and statistical expertise. P. S. and M. K. supervised the study. All authors contributed to the interpretation of the data and revision of the manuscript. M. K. had full access to all the data in the study and takes responsibility for the integrity of the data and the accuracy of the data analysis. None of the authors had any conflict of interest.

We are very grateful to the staff of the Research Institute of Child Nutrition for carrying out the anthropometric measurements and for collecting and coding the dietary records.

\section{References}

1. Malik VS, Schulze MB \& Hu FB (2006) Intake of sugar-sweetened beverages and weight gain: a systematic review. Am J Clin Nutr 84, 274-288.

2. Kant AK (2003) Reported consumption of low-nutrient-density foods by American children and adolescents: nutritional and health correlates, NHANES III, 1988 to 1994. Arch Pediatr Adolesc Med 157, 789-796.

3. Waijers PM, Feskens EJ \& Ocke MC (2007) A critical review of predefined diet quality scores. Br J Nutr 97, 219-231.

4. Kelder SH, Perry CL, Klepp KI, et al. (1994) Longitudinal tracking of adolescent smoking, physical activity, and food choice behaviors. Am J Public Health 84, 1121-1126.

5. Vartanian LR, Schwartz MB \& Brownell KD (2007) Effects of soft drink consumption on nutrition and health: a systematic review and meta-analysis. Am J Public Health 97, 667-675.

6. Duffey KJ \& Popkin BM (2006) Adults with healthier dietary patterns have healthier beverage patterns. $J$ Nutr 136, 2901-2907.

7. Alexy U, Sichert-Hellert W \& Kersting M (2002) Fifteen-year time trends in energy and macronutrient intake in German children and adolescents: results of the DONALD study. Br J Nutr 87, 595-604.

8. Bowman SA (1999) Diets of individuals based on energy intakes from added sugars. Fam Econ Nutr Rev 12, 31-38.

9. Farris RP, Nicklas TA, Myers L, et al. (1998) Nutrient intake and food group consumption of 10 -year-olds by sugar intake level: The Bogalusa Heart Study. J Am Coll Nutr 17, 579-585.

10. Forshee RA \& Storey ML (2001) The role of added sugars in the diet quality of children and adolescents. J Am Coll Nutr 20, 32-43.

11. Kroke A, Manz F, Kersting M, et al. (2004) The DONALD study. History, current status and future perspectives. Eur $J$ Nutr 43, 45-54.

12. Schofield WN (1985) Predicting basal metabolic rate, new standards and review of previous work. Hum Nutr Clin Nutr 39, Suppl 1, 5-41.

13. Sichert-Hellert W, Kersting M \& Schoch G (1998) Underreporting of energy intake in 1 to 18 year old German children and adolescents. Z Ernahrungswiss 37, 242-251.

14. Sichert-Hellert W, Kersting M, Chahda C, et al. (2007) German food composition database for dietary evaluations in children and adolescents. J Food Comp Anal 20, 63-70.

15. Gedrich K \& Karg G (2001) Dietary Habits of German vs NonGerman Residents in Germany. http://www.wlh.wi.tum.de/Res/ ICCAS2001/non-german.pdf

16. Gedrich K, Sichert-Hellert W, Kersting M, et al. (2006) Enhanced nutritional quality indices: evalution of two indices assessing the overall nutritional quality of diets. In Sixth International Conference on Dietary Assessment Methods, Copenhagen - Program and Abstracts, Poster P15-04. www.icdam6.dk/filer/Total\%20Abstracts.pdf $>21.08 .2008$.

17. Deutsche Gesellschaft für Ernährung (2000) Referenzwerte für die Nährstoffzufuhr (Reference Values for Nutrient Intake). Umschau/Braus: Frankfurt.

18. Spence JD, Bang H, Chambless LE, et al. (2005) Vitamin Intervention For Stroke Prevention trial: an efficacy analysis. Stroke 36, 2404-2409.

19. McCully KS (2007) Homocysteine, vitamins, and vascular disease prevention. Am J Clin Nutr 86, 1563S-1568S.

20. Cashman KD (2002) Calcium intake, calcium bioavailability and bone health. Br J Nutr 87, Suppl 2, S169-S177.

21. Harnack L, Stang J \& Story M (1999) Soft drink consumption among US children and adolescents: nutritional consequences. J Am Diet Assoc 99, 436-441.

22. Mrdjenovic G \& Levitsky DA (2003) Nutritional and energetic consequences of sweetened drink consumption in 6- to 13-yearold children. J Pediatr 142, 604-610.

23. Rodriguez-Artalejo F, Garcia EL, Gorgojo L, et al. (2003) Consumption of bakery products, sweetened soft drinks and yogurt among children aged 6-7 years: association with nutrient intake and overall diet quality. Br J Nutr 89, 419-429. 
24. Striegel-Moore RH, Thompson D, Affenito SG, et al. (2006) Correlates of beverage intake in adolescent girls: the National Heart. Lung, and Blood Institute Growth and Health Study. J Pediatr 148, 183-187.

25. Ballew C, Kuester S \& Gillespie C (2000) Beverage choices affect adequacy of children's nutrient intakes. Arch Pediatr Adolesc Med 154, 1148-1152.

26. Frary CD, Johnson RK \& Wang MQ (2004) Children and adolescents' choices of foods and beverages high in added sugars are associated with intakes of key nutrients and food groups. $J$ Adolesc Health 34, 56-63.

27. Marshall TA, Eichenberger Gilmore JM, Broffitt B, et al. (2005) Diet quality in young children is influenced by beverage consumption. J Am Coll Nutr 24, 65-75.

28. Forshee RA, Anderson PA \& Storey ML (2006) Changes in calcium intake and association with beverage consumption and demographics: comparing data from CSFII 1994-1996, 1998 and NHANES 1999-2002. J Am Coll Nutr 25, 108-116.

29. LaRowe TL, Moeller SM \& Adams AK (2007) Beverage patterns, diet quality, and body mass index of US preschool and school-aged children. J Am Diet Assoc 107, 1124-1133.

30. Forshee RA \& Storey ML (2006) Demographics, not beverage consumption, is associated with diet quality. Int J Food Sci Nutr 57, 494-511.

31. Alexy U, Sichert-Hellert W \& Kersting M (2002) Fortification masks nutrient dilution due to added sugars in the diet of children and adolescents. J Nutr 132, 2785-2791.
32. Libuda L, Alexy U, Sichert-Hellert W, et al. (2008) Pattern of beverage consumption and long-term association with body-weight status in German adolescents - results from the DONALD study. Br J Nutr 99, 1370-1379.

33. Madden JP \& Yoder MD (1972) Program evaluation: food stamps and commodity distribution in rural areas of central Pennsylvania. Penn Agr Exp Sta Bull 78, 1-119.

34. Guthrie HA \& Scheer JC (1981) Validity of a dietary score for assessing nutrient adequacy. J Am Diet Assoc 78, 240-245.

35. Davy BM, Harrell K, Stewart J, et al. (2004) Body weight status, dietary habits, and physical activity levels of middle school-aged children in rural Mississippi. South Med $J$ 97, $571-577$

36. Remer T, Fonteyn N, Alexy U, et al. (2006) Longitudinal examination of 24-h urinary iodine excretion in schoolchildren as a sensitive, hydration status-independent research tool for studying iodine status. Am J Clin Nutr 83, 639-646.

37. Mensink GBM, Heseker H \& Richter A (2007) Forschungsbericht Ernährungsstudie als KiGGS-Modul (EsKiMo) (in German). http://www.bmelv.de/SharedDocs/downloads/03Ernaehrung/EsKiMoStudie.html

38. Kersting M, Sichert-Hellert W, Alexy U, et al. (1998) Macronutrient intake of 1 to 18 year old German children and adolescents. $Z$ Ernahrungswiss 37, 252-259.

39. Alexy U, Kersting M, Sichert-Hellert W, et al. (1998) Energy intake and growth of 3- to 36-month-old German infants and children. Ann Nutr Metab 42, 68-74. 